\title{
Inhibition of NEDD8 and FAT10 ligase activities through the degrading enzyme NEDD8 ultimate buster 1: A potential anticancer approach (Review)
}

\author{
KA-LIONG TAN ${ }^{1,2}$ and FRANCESCO PEZZELLA ${ }^{1}$ \\ ${ }^{1}$ Nuffield Division of Clinical Laboratory Sciences, Radcliffe Department of Medicine, John Radcliffe Hospital, \\ Headington, Oxford OX3 9DU, United Kingdom; ${ }^{2}$ Faculty of Medicine \& Health Sciences, \\ Universiti Sains Islam Malaysia, Kuala Lumpur 55100, Malaysia
}

Received January 28, 2016; Accepted August 9, 2016

DOI: $10.3892 / 01.2016 .5232$

\begin{abstract}
The capabilities of tumour cells to survive through deregulated cell cycles and evade apoptosis are hallmarks of cancer. The ubiquitin-like proteins (UBL) proteasome system is important in regulating cell cycles via signaling proteins. Deregulation of the proteasomal system can lead to uncontrolled cell proliferation. The Skp, Cullin, F-box containing complex (SCF complex) is the predominant $\mathrm{E} 3$ ubiquitin ligase, and has diverse substrates. The ubiquitin ligase activity of the SCF complexes requires the conjugation of neural precursor cell expressed, developmentally down-regulated 8 (NEDD8) to cullin proteins. A tumour suppressor and degrading enzyme named NEDD8 ultimate buster 1 (NUB1) is able to recruit HLA-F-adjacent transcript 10 (FAT10)- and NEDD8-conjugated proteins for proteasomal degradation. Ubiquitination is associated with neddylation and FAT10ylation. Although validating the targets of UBLs, including ubiquitin, NEDD8 and FAT10, is challenging, understanding the biological significance of such substrates is an exciting research prospect. This present review discusses the interplay of these UBLs, as well as highlighting their inhibition through NUB1. Knowledge of the mechanisms by which NUB1 is able to downregulate the ubiquitin cascade via NEDD8 conjugation and the FAT10 pathway is essential. This will provide insights into potential cancer therapy that could be used to selectively suppress cancer growth.
\end{abstract}

Correspondence to: Mr. Ka-Liong Tan or Professor Francesco Pezzella, Nuffield Division of Clinical Laboratory Sciences, Radcliffe Department of Medicine, John Radcliffe Hospital, Headley Way, Headington, Oxford OX3 9DU, United Kingdom

E-mail: ka.tan@lincoln.ox.ac.uk

E-mail: francesco.pezzella@ndcls.ox.ac.uk

Key words: NEDD8, NUB1, FAT10, E3 ubiquitin ligases, cullins, proteasomal degradation, ubiquitin-like proteins

\section{Contents}

1. Overview of ubiquitin-proteasome system (UPS) and ubiquitin-like proteins (UBL) pathway as a therapeutic target

2. The NEDD8-conjugation (neddylation) pathway and the effect of neddylation on transcription factors

3. The FAT10-conjugation (FAT10ylation) pathway and its function

4. NEDD8 and FAT10 pathway perspectives

5. Conclusion

1. Overview of ubiquitin-proteasome system (UPS) and ubiquitin-like proteins (UBL) pathway as a therapeutic target

The UPS is important in regulating protein homeostasis in human cells through programmed degradation. The degradation of regulatory proteins can affect cell-cycle regulation, cell proliferation, intracellular signaling, DNA repair and apoptosis (1-4). As UPS degradation is a major clearance system associated with proteolysis within the cells, its deregulation can cause the pathogenesis of cancer and other diseases through the inappropriate loss of regulatory proteins or unintentional activation of certain specific signalling cascades. For instance, cancerous cells develop when cell-cycle controls break down, leading to unregulated cell proliferation. Cancer cells can also evade apoptosis induced by a number of different cellular stresses. Hence, an interruption to the normal regulation of the UPS could lead to abnormal cell proliferation.

The 26S proteasome is a protease complex capable of degrading polyubiquitinated proteins. The $26 \mathrm{~S}$ complex is composed of a barrel-shaped 20S proteasome core with a 19S regulatory particle at either or both of its ends. The $20 \mathrm{~S}$ proteasome contains the enzymatic active sites, whilst the $19 \mathrm{~S}$ regulatory particle helps to control access of ubiquitin-like protein (UBL)-conjugated substrates to the core. There are three proteasome active sites within the $20 \mathrm{~S}$ core, namely the caspase-like ( $(\beta 1)$, trypsin-like ( $\beta 2)$ and chymotrypsin-like ( $\beta 5)$ domains. These sites use an N-terminal threonine as the catalytic amino-acid residue $(5,6)$. 
Table I. First- and second-generation proteasome inhibitors.

\begin{tabular}{|c|c|c|}
\hline Drug & Company & Status \\
\hline \multicolumn{3}{|l|}{ First generation } \\
\hline Bortezomib & Millennium Pharmaceuticals, Inc. (Cambridge, MA, USA) & $\begin{array}{l}\text { FDA-approved for multiple } \\
\text { myeloma and relapsed } \\
\text { mantle cell lymphoma }\end{array}$ \\
\hline \multicolumn{3}{|l|}{ Second generation } \\
\hline Carfilzomib (Kyprolis) & Onyx Pharmaceuticals, Inc. (San Francisco, CA, USA) & $\begin{array}{l}\text { FDA-approved for multiple } \\
\text { myeloma }\end{array}$ \\
\hline Oprozomib (ONX0912) & Onyx Pharmaceuticals, Inc. (San Francisco, CA, USA) & Phase I \\
\hline Ixazomib citrate (MLN9708) & Millennium Pharmaceuticals, Inc. (Cambridge, MA, USA) & Phase I/II \\
\hline Marizomib (NPI-0052) & Nereus Pharmaceuticals (San Diego, CA, USA) & Phase I \\
\hline Delanzomib (CEP-18770) & Cephalon, Inc. (Frazer, PA, USA) & Phase I \\
\hline Calpeptin (IPSI-001) & Lanospharma Laboratories Co., Ltd. (Chongqing, China) & Phase I \\
\hline ONX0914 & Onyx Pharmaceuticals (San Francisco, CA, USA) & Phase I \\
\hline
\end{tabular}

FDA, Food and Drug Administration.

Ubiquitin (Ub) and UBLs share certain common structural elements, such as a three-dimensional structure called the Ub or $\beta$-grasp fold (7). The UBLs are a group of proteins encompassing neural precursor cell expressed, developmentally down-regulated 8 (NEDD8), small ubiquitin-like modifier $1 / 2 / 3$, interferon-stimulated gene 15 (ISG15), HLA-F-adjacent transcript 10 (FAT10), autophagy-related protein (ATG) 8 and ATG12, which conjugate to their targets in a manner similar to that of ubiquitination (8). Ub is a highly conserved protein of 76 amino acids that are able to attach to other proteins in a reversible fashion. There are three vital structural domains within $\mathrm{Ub}$ : i) the $\beta$-grasp fold, commonly found in all UBLs; ii) a C-terminal tail; and iii) seven lysine residues that correspond to polyubiquitin-linked chains (6). NEDD8 has distinctive functions in cells due to its structural differences, which mediate specialised interactions with target proteins compared with Ub. The crystal structure of NEDD8 is analogous to that of Ub, with the exception of two surface regions $(9,10)$. NEDD8 was initially discovered in fetal mouse brain (11) and can be found predominantly in adult tissues (11-13). NEDD8 and the neddylation pathway enzymes are overexpressed in human cancers (13-15). FAT10 is a 165 -amino acid protein that comprises two Ub-like domains with $29 \%$ identity and $36 \%$ homology to Ub at its N and C-termini, respectively (16). The protein is known to be involved in apoptosis, immune responses and cancer (16-18). Fig. 1A shows the domain structure of Ub, NEDD8 and FAT10.

The roles of UPS and UBL conjugation pathways in normal cell function and in disease has prompted the search for inhibitors that are able to selectively disrupt pathway function. Proteasome inhibitors have been synthesised to halt the function of the proteasomal activities. As mentioned, the proteasome has three active sites ( $\beta 1, \beta 2$ and $\beta 5)$, which utilise $\mathrm{N}$-terminal threonine as the catalytic amino acid residue (7). All UPS inhibitors were developed to covalently modify this threonine residue in order to block the enzyme's kinetics. The therapeutic value of UPS inhibition has been demonstrated with the proteasome inhibitor bortezomib (Velcade ${ }^{\circledR}$; Millennium Pharmaceuticals, Inc., Cambridge, MA, USA). Bortezomib is used in the treatment of patients suffering from multiple myeloma $(19,20)$ and mantle cell lymphoma (21).

As the first clinical proteasome inhibitor to target the UPS, bortezomib was approved in 2003. Several second-generation proteasome inhibitors are currently in development, such as carfilzomib, oprozomib, ixazomib citrate, marizomib and delanzomib. In comparison with UPS inhibition, blocking of UBL pathways may provide a more specific effect by targeting the substrate proteins. UPS inhibition is a common step in blocking the degradation of a broader range of substrates. For instance, inhibition of E1-activating enzymes may be achieved through covalent inactivation (e.g. PYR-41) (22) and adduct formation (e.g. MLN4924) (23). Blocked E2 interactions offer a more selective inhibition (e.g. synthetic peptide UBC12N26). Recent research has frequently focussed on targeting deubiquitinating enzymes (DUBs), as this class of proteins are capable of reversing the action of the Ub conjugation cascade. Table I summarises the current clinical development of second-generation proteasome inhibitors, and Table II lists the E1/2/3 inhibitors. Furthermore, a NEDD8-activating enzyme (NAE) inhibitor, MLN4924, which targets the NEDD8 pathway, appears to be a potentially important anticancer strategy (24). The development of DUB inhibitors is more recent compared with that of the proteasome and E1/2/3 inhibitors. To the best of our knowledge, no DUB inhibitors have entered clinical trials.

NEDD8 ultimate buster 1 (NUB1), a NEDD8- and FAT10-degrading enzyme, and approaches to anticancer therapy. NUB1 is an interferon (IFN)-inducible protein of $69 \mathrm{kDa}$, composed of 601 amino acids. It also has a splice variant, NUB1L, which possesses an extra 14 amino acids that encode an additional Ub-associated (UBA) domain (Fig. 1B). NUB1 proteins can recruit FAT10- and NEDD8-conjugated proteins to the proteasome for degradation and negatively 
Table II. A summary of small molecule inhibitors targeting E1s, E2s and E3s.

\begin{tabular}{llll}
\hline Drug & Target & & Company \\
\hline PYR-41 & E1 & Millennium Pharmaceuticals, Inc. (Cambridge, MA, USA) & Status \\
MLN4924 & E1 & Millennium Pharmaceuticals, Inc. (Cambridge, MA, USA) & N/A \\
CC0651 & E2 & N/A & N/A \\
NSC697923 & E2 & N/A & N/A \\
Nutlin & E3 & Roche Products Limited (Pharmaceuticals) (Welwyn Garden City, UK) \\
MI-773 & E3 & Sanofi S.A. (Gentilly, France) & Phase I \\
CGM097 & E3 & Novartis International AG (Basel, Switzerland) & Phase I \\
\hline
\end{tabular}

N/A, not applicable.

A

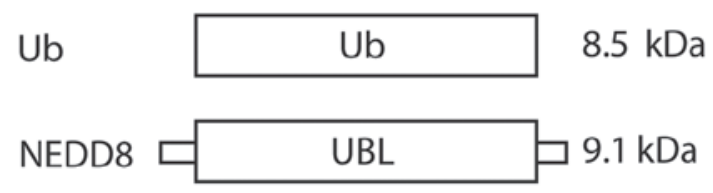

FAT10 UBL UBL $18.5 \mathrm{kDa}$

B

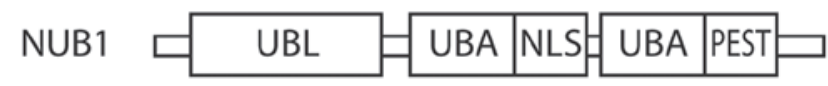

Figure 1. Structural representation of (A) Ub, NEDD8 and FAT10, and (B) NUB1. Ub, ubiquitin; NEDD8, neural precursor cell expressed, developmentally down-regulated 8; FAT10, HLA-F-adjacent transcript 10; NUB1, NEDD8 ultimate buster 1; UBL, ubiquitin-like domain; UBA, ubiquitin-associated; NLS, nuclear localisation signal; PEST, proline-enriched glutamic acid, serine and threonine domain.

regulate the NEDD8-conjugation system (25-29). The NUB1 proteins have been observed in various types of cancer cells, including cervical adenocarcinoma, rectal adenocarcinoma, neuroblastoma, malignant lymphoma and renal cell carcinoma (RCC) (26). Upregulated NUB1 expression has been linked to IFN $\alpha$-induced antimitogenic actions. Additionally, NUB1 has demonstrated anticancer properties in RCC cell lines, where it was involved in apoptosis and S-phase transition through its action on p27 and cyclin E (30,31). Upregulation of NUB1 effectively inhibits the proliferation of IFN $\alpha$-resistant RCC cells (31).

NUB1 protein has been reported to play a role in Huntington's disease (32) and congenital amaurosis (33). In cancers, NUB1 is an attractive candidate for inhibition of $\mathrm{p} 27^{\mathrm{KIP} 1}$ and $\mathrm{p} 21^{\mathrm{CIP} 1}$ via the regulation of the Skp, Cullin, F-box-containing $(\mathrm{SCF})^{\mathrm{SKP} 2}$ ligase activity (31). The upregulated $\mathrm{p} 21^{\mathrm{CIP} 1}$ in NUB1-knockdown cancer cells is thought to be promising in directing the cells to senescence. NUB1 protein was reported to be a tumour suppressor as it exerts growth inhibition during its overexpression; upon IFN $\alpha$ treatment, overexpressed NUB1 induced apoptosis in IFN $\alpha$-resistant A498 cells (31). However, its general lack of enzymatic activities makes NUB1 less suitable for small molecule inhibition (32). Thus, the low-molecular-weight proteins FAT10 and NEDD8 could be key to developing novel strategies in anticancer therapy, as they interact with NUB1 (34). The current review focuses on the relevance of NUB1 protein in NEDD8 and FAT10 conjugation in cancers, and the potential for targeting it as a novel therapeutic approach.

\section{The NEDD8-conjugation (neddylation) pathway and the effect of neddylation on transcription factors}

The UBL enzymatic cascade scheme that results in UBL conjugation and protein degradation involves several distinct steps. Each step requires different classes of enzyme, as shown in Table III.

Neddylation is a post-translational modification process that conjugates NEDD8 to its target proteins, in a process that is analogous to that observed for ubiquitination. However, the neddylation process uses a distinct E1 and E2 enzyme reaction scheme $(5,8,11,35,36)$ (Table III). Fig. 2A summarises the NEDD8 conjugation and deconjugation steps $(34,37)$. The C-terminal glycine of NEDD8 is adenylated by an E1 NEDD8-activating enzyme (NAE), a heterodimer composed of amyloid- $\beta$ precursor protein-binding protein 1 (APPBP1) and Ub-like modifier-activating enzyme (UBA) 3. NEDD8 is covalently conjugated to the NAE via a thiolester linkage (38). The activated NEDD8 is consecutively transferred to the E2 NEDD8-conjugation enzyme and then to the specific substrates (i.e. cullin proteins) via an isopeptide bond (39). The RING-box protein (RBX) 1/ROC1, mouse double minute 2 homolog (MDM2), F-box protein 11 and c-Cbl proteins are neddylated in the same way (39).

There are two NEDD8-specific E2-conjugating enzymes, namely ubiquitin conjugating enzyme E2 (UBE2)M (also known as UBC12) and UBE2F. These E2 enzymes act to 
Table III. Overview of the enzymatic cascade involved in UBL conjugation.

\begin{tabular}{llll}
\hline UBL & \multicolumn{1}{c}{ Ub } & NEDD8 & FAT10 and Ub \\
\hline E1-activating enzymes & UAE & APPBP1-UBA3 heterodimer & UBA6 \\
E2-conjugating enzymes & UBCs & UBE2M and UBE2F & USE1 \\
E3 ligases & Ub E3 ligases & NEDD8 E3 Ligases & N/A \\
Substrates & $1,000 \mathrm{~s}$ & $200 \mathrm{~s}$ & N/A \\
\hline
\end{tabular}

UBL, ubiquitin-like protein; Ub, ubiquitin; NEDD8, neural precursor cell expressed, developmentally down-regulated 8; FAT10, HLA-F-adjacent transcript 10; UAE, ubiquitin-activating enzymes; APPBP1, amyloid- $\beta$ precursor protein-binding protein 1; UBA, ubiquitin-like modifier-activating enzyme; UBCs, Ub-conjugating enzymes; UBE2, ubiquitin-conjugating enzymes E2; USE1, UBA6-specific E2 enzyme; N/A, not applicable.

A

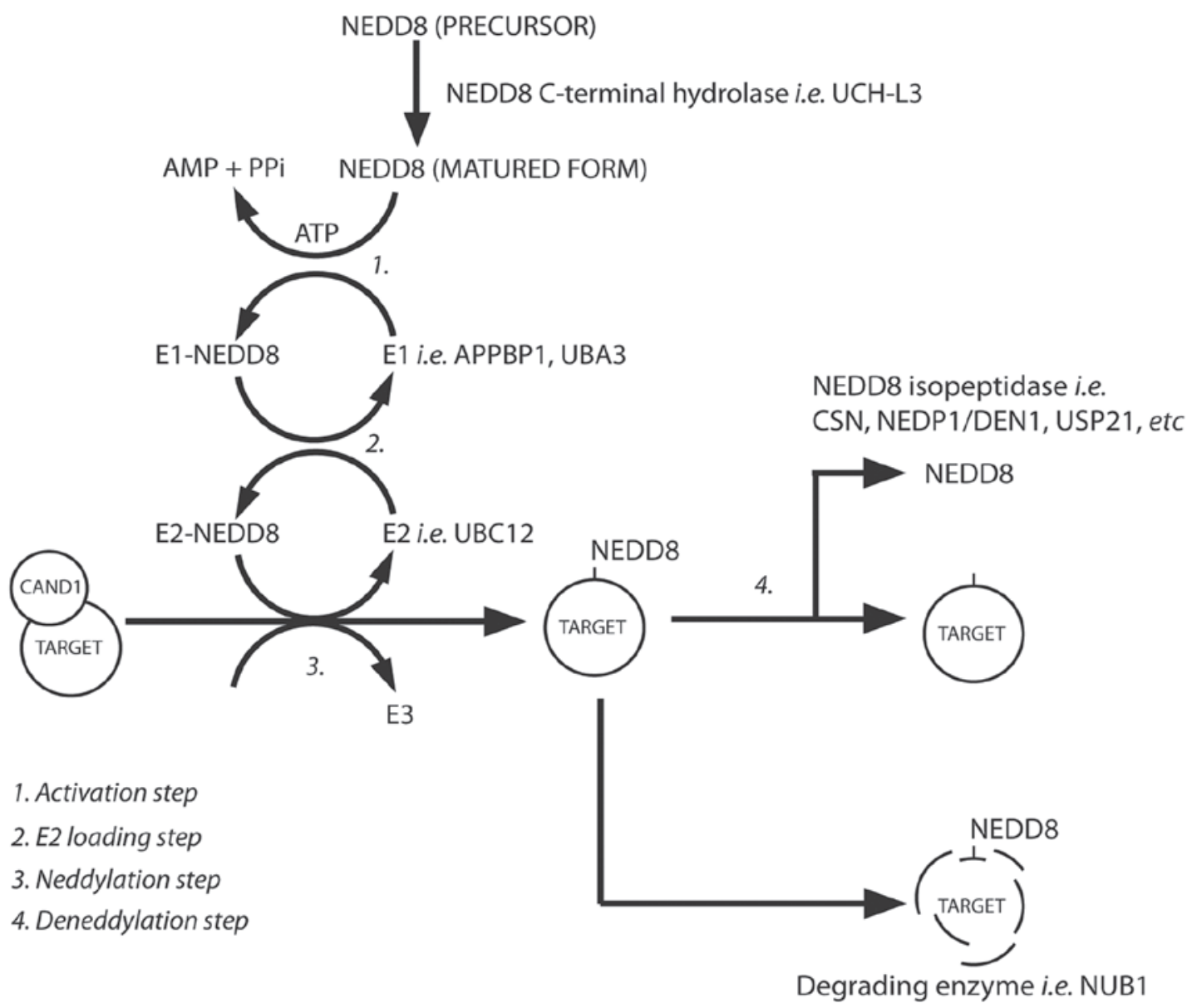

B

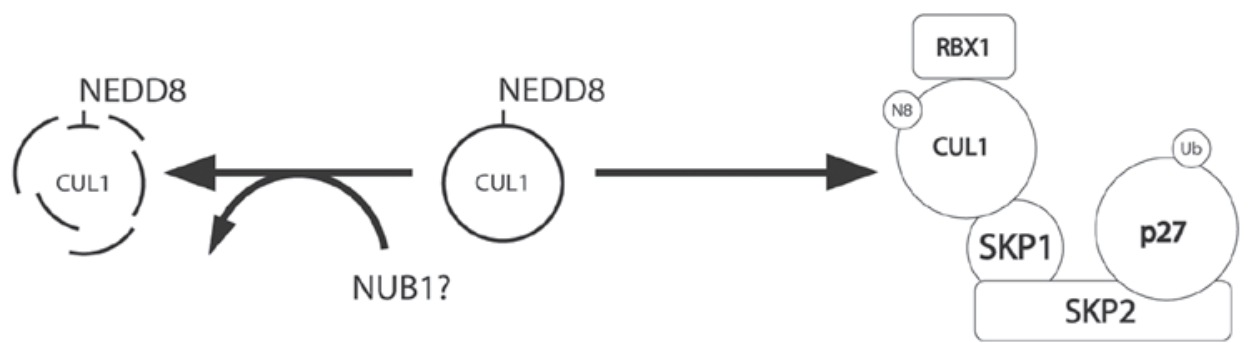

Figure 2. (A) NEDD8 conjugation pathway. Schematic summary of the main steps of the neddylation pathway [modified from Rabut and Peter, 2008 (37); Tanaka et al, 2012 (34)]. (B) Neddylated CUL1 locks the SCF complex with phosphorylated p27 and cyclin E [as suggested by Bornstein et al, 2006 (30) and Tanaka et al, 2012 (34)]. CAND1, cullin-associated and neddylation-dissociated 1; AMP, adenosine monophosphate; PPi, anion P2O74-; ATP, adenosine triphosphate; UCH-L3, ubiquitin C-terminal hydrolase isozyme L3; APPBP1, amyloid- $\beta$ precursor protein binding protein 1 ; DEN1, deneddylase 1 , CSN, COP9 signalosome; NEDP1, NEDD8-specific protease 1; NUB1, NEDD8 ultimate buster 1; USP21, ubiquitin specific peptidase 21, CUL1, cullin 1; SKP1, S-phase kinase-associated protein 1; SKP2, S-phase kinase-associated protein 2; RBX1, ring-box 1, E3 ubiquitin protein ligase; SCF, Skp, cullin, F-box-containing complex. 
transfer NEDD8 to its target protein through E3 enzymes. It has been reported that all NEDD8 E3 enzymes can function as Ub E3 enzymes. The predominant NEDD8 E3 ligases are the RING subunits RBX1 and RBX2 (38,40-43). Meanwhile, the non-RBX family NEDD8 E3 ligases include c-CBL, ring finger protein 111, MDM2 and inhibitor of apoptosis 1 (44).

Of the numerous NEDD8 substrates, neddylation has been best described in the cullin family (45). In this mechanism, NEDD8 from the E2 cysteine active site is transferred onto a lysine residue in the $\mathrm{N}$-terminus of the target proteins (Fig. 2A) (46). Cullin neddylation is further mediated by defective in cullin neddylation protein 1-like proteins (44). It was reported that RING E3 ligases could neddylate the same substrate on multiple lysine residues (44). However, the interaction between non-RBX RING E3 ligase and E2 enzymes remains to be elucidated.

NEDD8-conjugated substrates are deneddylated by various proteins that include COP9 signalosome (CSN), NEDD8-specific protease 1 (NEDP1/DEN1) and ubiquitin specific peptidase 21 (37,47-51). Neddylation may be inhibited by cullin-associated and neddylation-dissociated 1 through its direct binding to cullins $(52,53)$. NEDD8 and neddylated substrates are recruited by NUB1 for proteasomal degradation (25,26) (Fig. 2A). In the G1-S-phase transition, Bornstein et al (30) demonstrated how neddylated cullin 1 cooperatively activates the $\mathrm{SCF}^{\mathrm{SKP} 2} \mathrm{Ub}$ ligase complex, which results in p27 degradation (Fig. 2B). Furthermore, previous studies found that cullin neddylation increased the Ub E3 ligase activity of the SCF complex (Fig. 3) $(46,54)$.

NEDD8 is negatively regulated by NUB1, which links the UBLs to the $26 \mathrm{~S}$ proteasome for further UPS degradation. Reports have described that NUB1 is able to recruit NEDD8 and NEDD8-conjugated proteins to the proteasome for degradation, and this may modulate the cell-cycle profile in response to stresses (34). The capability of NEDD8 to activate the Ub E3 ligase-SCF complex (by covalent binding to cullins) adds further complexity to the ubiquitination machinery $(11,55-59)$. Therefore, validation of NEDD8 targets would allow identification of genuine NEDD8 substrates.

Challenges in identifying physiological neddylation targets. Hjerpe et al (45) demonstrated that NEDD8 and Ub cascades are independent of one another during normal cellular homeostasis. NEDD8 conjugation onto Ub substrates through the Ub cascade has a spurious role in normal physiological conditions. The single amino acid change in the C-terminus of NEDD8 compared to Ub, from Arg72 to Ala72, confers the specificity between these two UBLs (44). This ensures that the correct UBL is passed to the appropriate E2 enzyme, E3 enzyme and the substrate respectively (Table III). However, when NEDD8 is in excess, the NEDD8 E1 enzyme UBA1 can activate NEDD8, which is then transthiolated to Ub E2 enzymes. This phenomenon results in the neddylation of Ub-specific substrates $(10,45)$. NEDD8 can form NEDD8 chains or mixed Ub-NEDD8 chains $(39,60)$. An increase of NEDD8 over Ub, as a result of cellular stresses, cellular diversity or pathological conditions, could exert different effects on neddylated substrates (44). This raises concerns, since the majority of research performed to date to identify neddylated substrates in cells relies on the overexpression of NEDD8; as this would cause an imbalance

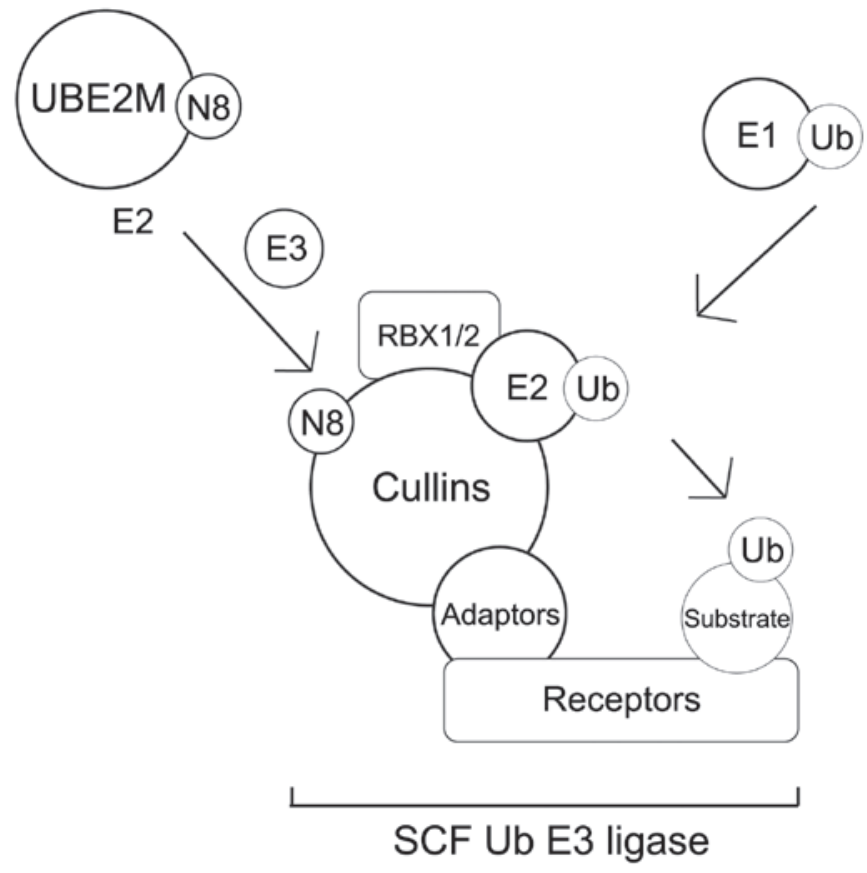

Figure 3. NEDD8 conjugation and ubiquitination pathway through cullin proteins. UBE2M, ubiquitin-conjugating enzyme E2M; RBX1/2, ring-box 1; Ub, ubiquitin; NEDD8, neural precursor cell expressed, developmentally down-regulated 8; SCF, Skp, cullin, F-box-containing complex.

between cellular NEDD8 and Ub levels, it could result in the aberrant neddylation of proteins via the Ub pathway (45).

Enchev et al (44) therefore revised and proposed a set of criteria to define the search for physiological neddylation targets: A neddylation substrate must demonstrate the covalent attachment of NEDD8 through the carboxyl-terminal glycine to the lysine residue of the substrates; and the neddylation must be detected under homeostatic conditions under endogenous NEDD8 levels and substrate expression. The NAE inhibitor MLN4924 should be incorporated into the study, as it blocks cullin neddylation but not ubiquitination (44). It remains optional to examine the possible NEDD8 E2 and E3 enzymes $(44,45)$. It is also advisable to look at the regulation and biological consequences of neddylation (44). In endogenous protein experiments, immunoprecipitation with specific antibodies is a recommended approach (44). Genome editing techniques, such as a CRISPR/cas9 approach, may be used to introduce affinity-tagged versions of a particular gene product (44). The NEDD8 substrate should also be confirmed using mass spectrometry, using LysC protease as the cleavage enzyme, as it can discriminate between Ub, NEDD8 and ISG15 conjugates (61). Mass spectrometry can also be used to determine the site of the neddylated Lys residue, and the type of NEDD8 chains that are formed. The neddylated Lys residue needs further study if it is also targeted by Ub. The relative abundance of Ub, NEDD 8 and FAT10 must be examined for its physiological relevance (44). A mutant form of the substrate that can no longer be neddylated must also be included to serve as a negative experimental control (44).

Overexpression of NEDD8 and the aberrant activation of the neddylation pathway and cullin-RING Ub ligase (CRL) activity can drive the progression of cancers $(4,13)$, inflammatory and autoimmune diseases (7). Mainstream research 
focuses on the effects of CRL inhibition, neddylation and deneddylation. The small-molecule NAE inhibitor MLN4924 is undergoing clinical trials. MLN4924 is an analog of adenosine monophosphate that competitively binds to the enzymatic pocket of NAE. This small molecule therefore inhibits neddylation and CRL activity. MLN4924 treatment causes DNA replication by stabilising chromatin licensing and DNA replication factor 1, a DNA replication licensing factor and CRL substrate. MLN4924-treated cells accumulate DNA damage due to DNA repair failure, leading to apoptosis (62) or senescence (19). Neddylation is able to inhibit the transcriptional activity of the tumour suppressors p53 and p73, and to stabilise Hu-antigen R (63), cell division cycle 6 and hypoxia-inducible factors (64). One of the important outcomes of MLN4924 treatment is that it causes the cancer cells to undergo apoptosis and senescence (44).

Transcriptional regulation via the neddylation of transcription factors. Several studies have suggested that neddylation of transcription factors can lead to the suppression of their transcriptional activity $(44,65)$.

E2F transcription factor 1 (E2F1). Neddylation of E2Fs reduces their transcriptional activity $(66,67)$. E2F1 was shown to be neddylated in the DNA-binding domain and its protein levels reduced following neddylation (67). DEN1 deneddylates E2Fs and consequently activates E2F-mediated transcription. DNA damage promotes the expression of DEN1, which subsequently deneddylates E2F and causes its stabilisation (68). Neddylation specifically regulates a subset of E2F target genes; for example, E2F1 deneddylation upon DNA damage triggers the transcription of proapoptotic factors (66).

p53 and p73. p53 acts by inhibiting cell cycle progression or triggering senescence or apoptosis (33). It is inhibited by the RING-domain E3 ligase MDM2, which targets ubiquitinated p53 for degradation. MDM2 is able to neddylate p53 and inhibit its transcriptional activity (33). The neddylated p53 is further recruited by NUB1, leading to its inactivation (69). p73 that is neddylated by MDM2 undergoes cytoplasmic relocalisation and downregulation of transcriptional activity (70). In addition, the Ub E3 ligase SKP1-CUL1-F-box protein 11 (SCF $\left.{ }^{\mathrm{Fbox} 11}\right)$ may neddylate $\mathrm{p} 73$ and downregulate its transcriptional activities (71).

Nuclear factor $\kappa B(N F-\kappa B)$. When extracellular signaling is absent, $N F-\kappa B$ is distributed in the cytoplasm and inhibited by inhibitor of $\mathrm{NF}-\kappa \mathrm{B}(\mathrm{I} \kappa \mathrm{B})$ family members. Upon stimulation by proinflammatory cytokines [such as tumour necrosis factor (TNF)], IкB kinases (IKKs) $-\alpha,-\beta$ and $-\gamma$ phosphorylate $\mathrm{I} \kappa \mathrm{B}$, which is then ubiquitylated and targeted for degradation by $\mathrm{SCF}^{\beta T \mathrm{TCP}}$. Under the same conditions, IKK $\gamma$ was reported to be neddylated and degraded by the proteasome, which reduces $N F-\kappa B$ activation and inhibits NF- $\kappa \mathrm{B}$ activity in gastrointestinal neoplasia (72). Therefore, neddylated IKK $\gamma$ may exert a tumour suppressor function.

Amyloid precursor protein intracellular domain (AICD). Amyloid precursor protein is cleaved by secretase to become amyloid- $\beta$ peptide and AICD. AICD is a component of a transcription factor complex with amyloid- $\beta$ (A4) precursor protein-binding family B member 1 (FE65) and
TAT-interactive protein 60 (73). Neddylated AICD blocks its interaction with FE65 and prevents the formation of the transcription factor complex, thereby reducing its transcriptional activity $(2,74)$. Thus, neddylated AICD inhibits the transcription of downstream targets.

\section{The FAT10-conjugation (FAT10ylation) pathway and its function}

FAT10 was discovered by Sherman Weissman in 1996 (5). Due to the poor solubility of the protein at high concentrations, the structure of FAT10 protein was only recently defined (75). FAT10 consists of two $\beta$-grasp fold domains connected by a short linker (75). FAT10 protein was found to be expressed predominantly in immune tissue, including the thymus, lymph nodes and spleen (76-78). Its expression is stimulated by proinflammatory cytokines, namely IFN $\gamma$ and TNF $\alpha$ (79). FAT10 protein is found in mature dendritic cells and it demonstrates oncogenic characteristics; ectopic expression of FAT10 causes malignant transformation and promotes tumour growth (80), and it is known to be upregulated in several tumour types, including liver and colon tumours $(18,81)$.

FAT10 shares the same E1 and E2 enzymes with the Ub conjugation pathway. The FAT10 E1 enzyme UBA6 is able to activate Ub and FAT10 (29,82-84). The adenylation and transthiolation reactions of FAT10 are kinetically slower than those for Ub. UBA6 protein is thought to be the only FAT10 E1 enzyme in cells, since UBA6 knockdown can effectively abolish the formation of FAT10-conjugates in vitro $(84,85)$. Similarly, UBA6-specific E2 enzyme (USE1) is the only UBA6-specific E2 enzyme discovered to be involved in FAT10 conjugation, although it also functions in a similar fashion to the conjugation of Ub (83). USE1 may only bind to activated Ub from UBA6, not UBE1 (83).

Little is currently known about FAT10, and research to identify possible FAT10 E3 ligases and deconjugating enzymes is ongoing. One study demonstrated that ectopically expressed FAT10 was not degraded over time, suggesting the possibility that a group of FAT10-deconjugating enzymes may not exist (86). It is believed that FAT10 is capable of promoting its own proteasomal-dependent degradation without the aid of deconjugating enzymes (86). FAT10-conjugated proteins were found to have a reduced half-life, similar to that observed for Ub-conjugated proteins (21). Conversely, it was demonstrated that FAT10-conjugated p62 accumulated under proteasome inhibition (79).

The interferon-inducible protein NUB1 interacts with FAT10 non-covalently (25), and significantly accelerates the degradation of FAT10 by the proteasome (25). NUB1 binds to the proteasome subunit S5a (28), and also to FAT10 via its three C-terminal UBA domains (Fig. 4) (87). NUB1 is also able to interact with the von Willebrand A (VWA) domain of RPN10 (S5a), one of the subunits of the $26 \mathrm{~S}$ proteasome $(25,28)$. The degradation of FAT10 is accelerated further by NUB1 splicing variant, NUB1L, which is able to bind to regulatory particle non-ATPase (RPN)10 in addition to the 19S regulator subunit, RPN1 (S2) (87).

The 26S proteasome subunit Rpn10 (S5a) is the docking site for FAT10, NUB1L and polyubiquitin. Ub interaction motifs 1 and 2 of Rpn10 are bound by lysine 48-linked polyubiquitin 


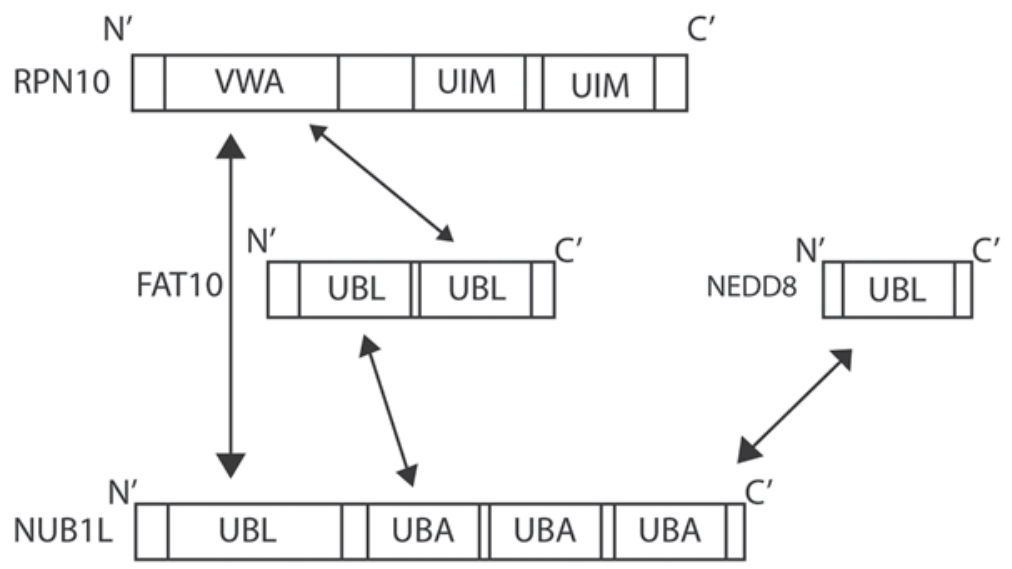

Figure 4. Domain structure of NUB1L, FAT10 and RPN10 and their interacting domains [Hipp et al (87); Kamitani et al (25); Tanji et al (28)]. RPN10, regulatory particle non-ATPase 10; FAT10, HLA-F-adjacent transcript 10, NUB1L, NEDD8 ultimate buster 1 long isoform; UBL, ubiquitin-like domain; UBA, ubiquitin-associated; UIM, ubiquitin-interacting motif; VWA, von Willebrand A motif.

chains. FAT10 is able to target substrate proteins to the proteasome independently of poly-FAT10ylation. FAT10 interacts directly with the VWA domain of RPN10, and no ubiquitination is required (28). The co-expression of NUB1L has been shown to accelerate the degradation of FAT10, suggesting a preference for proteasomal degradation (28).

Substrates of FAT10 conjugation. The biological function of FAT10 remains poorly understood. FAT10 overexpression has been demonstrated to induce apoptosis in mouse fibroblasts (67), HeLa cells (68) and renal tubular epithelial cells (70). FAT10 is synergistically induced by IFN $\gamma$ and TNF- $\alpha$, which leads to the induction of apoptosis (25). Several FAT10-interacting proteins have been identified, and are summarised in the following paragraphs.

The inflammatory mediator leucine-rich repeat Fli-I-interacting protein 2 (LRRFIP2) is covalently modified by FAT10 (6). LRRFIP2 positively regulates the activity of $\mathrm{NF}-\kappa \mathrm{B}$ in the inflammatory response mediated by toll-like receptor (TLR)4 (6). FAT10ylation of LRRFIP2 hinders its recruitment to the plasma membrane, which results in the inhibition of lipopolysaccharides (LPS)/TLR4-mediated $N F-\kappa B$ activation (6). This consequently leads to the reduced expression of NF- $\mathrm{B}$-responsive genes, including apoptosis inhibitors (6). Overexpression of FAT10 can induce apoptosis, causing FAT10 ${ }^{\text {NULL }}$ mice to be hypersensitive to LPS challenge due to NF- $\mathrm{NB}$ inhibition (33). However, FAT10 was observed to protect leukocytes in the spleen, thymus and bone marrow from apoptosis in a mouse model (33). In another study, the colon cancer cell line HCT116 was protected from TNF- $\alpha$-induced apoptosis in the presence of FAT10 (88). The induction of apoptosis by FAT10 is therefore cell type-specific; however, the mechanisms involved remain unknown.

Mitotic arrest-deficient 2 (MAD2), a spindle assembly checkpoint protein, binds to FAT10 protein non-covalently (33). In prometaphase, overexpressed FAT10 in HCT116 cells was found to reduce the localisation of MAD2 at the kinetochore $(88,89)$. Ren et al (88) reported that TNF- $\alpha$-induced upregulation of FAT10 also delocalised MAD2 from kinetochores in a similar way and accelerated cell mitosis. The mis-segregation of chromosomes was shown to be abolished when FAT10 levels were reduced by siRNA (89). Hence, FAT10 is considered to cause mis-segregation of chromosomes during cell division $(88,89)$.

FAT10 has been found to be highly expressed in colorectal, ovarian, hepatocellular and uterine carcinomas, suggesting that FAT10 expression may promote oncogenesis (89). A study found that $72 \%$ of hepatocellular carcinoma and $53 \%$ of colon carcinoma tissues overexpressing FAT10 also expressed the IFN $\gamma /$ TNF- $\alpha$-dependent immunoproteasome subunit low molecular mass protein 2 (90), suggesting that the pro-inflammatory cytokine response may be responsible for FAT10 overexpression in carcinoma tissues.

Autophagy adaptor p62 or sequestosome-1 protein can regulate aggresome formation, which protects cells from aggregation-prone protein-induced toxicity (79). FAT10ylated p62 tends to be proteasomally degraded (79). A previous study revealed that FAT10 expression induced by pro-inflammatory cytokines leads to a decrease in endogenous p62 (79). FAT10 was found to be transported by histone deacetlyase 6 along microtubules into aggresomes, causing p62 degradation (79). Under pathological conditions, p62 is localised in aggresomes along with the aggregated proteins found in neuronal diseases, including Alzheimers (91). The impact of FAT10 on P62-induced pathogenesis remains unresolved. However, there is no evidence that FAT10ylated p62 has a role in autophagic pathways (91).

\section{NEDD8 and FAT10 pathway perspectives}

The SCF Ub E3 ligases have been shown to be deregulated in various cancers; this results in unlimited cell proliferation and carcinogenesis via accumulation of their substrate proteins (34). Consequently, the E3 ligases are the subject of research into potential strategies for anticancer therapy (34). It is believed that the NEDD8-Ub-SCF complexes and the NEDD8-FAT10-degrading enzyme NUB1 are potential candidates for therapy (Fig. 2B) (34).

The search for neddylation targets requires further experimental validation. The conventional ectopic overexpression of UBLs is thought to lead to false positive conjugation of substrates (45). However, genome editing techniques, such as CRISPR-Cas9 technology, could overcome this, as it permits the neddylated substrates to be examined endogenously (44). 
In addition to NEDD8 and Ub chain formation, proteomic studies have reported phosphorylation, acetylation and succinylation sites on NEDD8. The functional significance of this observation remains unknown. There is a general lack of information on non-cullin protein neddylation under homeostatic conditions. Furthermore, the physiological relevance of several reported NEDD8 substrates, including p62/sequestosome, remains unknown. Whether neddylation is functionally distinct from ubiquitination is a question that remains unresolved. For example, polyneddylation and polyubiquitination at DNA damage sites or in response to other stress conditions are functionally redundant, and NEDD8 and $\mathrm{Ub}$ may be recognised by the same interaction motifs. However, in certain circumstances, such as the neddylation or ubiquitination of TGF $\beta$ RII, these two modifications can elicit distinct biological responses. Efforts are clearly needed to identify and characterise NEDD8-interacting domains and proteins.

NUB1 proteins cause the degradation of FAT10- and NEDD8-conjugated targets. Their expression regulates NEDD8- and FAT10-based signalling in response to cellular stresses $(34,45)$. However, the structural mechanisms of NUB1 protein and its clinical relevance in UBL pathways remain to be explored. Hosono et al (31) found that overexpression of NUB1 inhibits cell growth, and the same study demonstrated lower NUB1 mRNA expression in IFN $\alpha$-sensitive 4THUR cells. The same study highlighted that NUB1 is not induced in IFN $\alpha$-resistant cells, although transiently expressed NUB1 sensitised the same cells and induced apoptosis (31). Therefore, killing IFN $\alpha$-resistant cells by increasing NUB1 activity is a potential strategy (31).

FAT10 research is still in an early stage and the biological consequences of FAT10ylation are poorly described. De-FAT10ylating enzymes are under active investigation as drug targets in the pharmaceutical industry at present, based on the fact that a number of putative FAT10 targets are oncogenes or inhibitors of apoptosis. Future works should focus on the FAT10-modulated proteasome system and mechanisms of cytokine-induced reactions.

\section{Conclusion}

Experimental studies have demonstrated that negative regulation of the Ub, NEDD8 and FAT10-conjugation pathways have great potential in the context of cancer suppression. SCF complexes are often deregulated in cancer and could be modulated through manipulation of cullin neddylation. Neddylation and FAT10ylation inhibitors have recently been developed as a novel class of anticancer agent. These compounds are expected to exhibit better specificity for cancer cells and have reduced toxicity. Degrading enzymes, such as CSN and NUB1/NUB1L, are attractive candidates for the inhibition of Ub, NEDD8 and FAT10-ligase activities (34). These are expected to provide new strategies in anticancer therapy.

\section{Acknowledgements}

Mr. Ka-Liong Tan is financially supported by the Academic Staff Training Scheme (SLAI scheme), Ministry of Education, Malaysia.

\section{References}

1. Ciechanover A: The ubiquitin-proteasome pathway: On protein death and cell life. EMBO J 17: 7151-7160, 1998.

2. Ciechanover A and Schwartz AL: The ubiquitin-proteasome pathway: The complexity and myriad functions of proteins death. Proc Natl Acad Sci USA 95: 2727-2730, 1998.

3. Hershko A: The ubiquitin system for protein degradation and some of its roles in the control of the cell division cycle. Cell Death Differ 12: 1191-1197, 2005.

4. Hershko A and Ciechanover A: The ubiquitin system. Annu Rev Biochem 67: 425-479, 1998.

5. Liu YC, Pan J, Zhang C, Fan W, Collinge M, Bender JR and Weissman SM: A MHC-encoded ubiquitin-like protein (FAT10) binds noncovalently to the spindle assembly checkpoint protein MAD2. Proc Natl Acad Sci USA 96: 4313-4318, 1999.

6. Gunawardena HP, Huang Y, Kenjale R, Wang H, Xie L and Chen $\mathrm{X}$ : Unambiguous characterization of site-specific phosphorylation of leucine-rich repeat Fli-I-interacting protein 2 (LRRFIP2) in Toll-like receptor 4 (TLR4)-mediated signaling. J Biol Chem 286: 10897-10910, 2011.

7. Hochstrasser M: Origin and function of ubiquitin-like proteins. Nature 458: 422-429, 2009.

8. Bawa-Khalfe T and Yeh ET: SUMO losing balance: SUMO proteases disrupt SUMO homeostasis to facilitate cancer development and progression. Genes Cancer 1: 748-752, 2010.

9. Rao-Naik C, delaCruz W, Laplaza JM, Tan S, Callis J and Fisher AJ: The rub family of ubiquitin-like proteins. Crystal structure of Arabidopsis rub1 and expression of multiple rubs in Arabidopsis. J Biol Chem 273: 34976-34982, 1998.

10. Whitby FG, Xia G, Pickart CM and Hill CP: Crystal structure of the human ubiquitin-like protein NEDD8 and interactions with ubiquitin pathway enzymes. J Biol Chem 273: 34983-34991, 1998.

11. Kamitani T, Kito K, Nguyen HP and Yeh ET: Characterization of NEDD8, a developmentally down-regulated ubiquitin-like protein. J Biol Chem 272: 28557-28562, 1997.

12. Kim DY, Kwon E, Hartley PD, Crosby DC, Mann S, Krogan NJ and Gross JD: CBF $\beta$ stabilizes HIV Vif to counteract APOBEC3 at the expense of RUNX1 target gene expression. Mol Cell 49: 632-644, 2013.

13. Hori T, Osaka F, Chiba T, Miyamoto C, Okabayashi K, Shimbara N, Kato S and Tanaka K: Covalent modification of all members of human cullin family proteins by NEDD8. Oncogene 18: 6829-6834, 1999.

14. Salon C, Brambilla E, Brambilla C, Lantuejoul S, Gazzeri S and Eymin B: Altered pattern of Cul-1 protein expression and neddylation in human lung tumours: Relationships with CAND1 and cyclin E protein levels. J Pathol 213: 303-310, 2007.

15. Chairatvit K and Ngamkitidechakul C: Control of cell proliferation via elevated NEDD8 conjugation in oral squamous cell carcinoma. Mol Cell Biochem 306: 163-169, 2007.

16. Raasi S, Schmidtke G and Groettrup M: The ubiquitin-like protein FAT10 forms covalent conjugates and induces apoptosis. J Biol Chem 276: 35334-35343, 2001.

17. Fan W, Cai W, Parimoo S, Schwarz DC, Lennon GG and Weissman SM: Identification of seven new human MHC class I region genes around the HLA-F locus. Immunogenetics 44: 97-103, 1996.

18. Lee CG, Ren J, Cheong IS, Ban KH, Ooi LL, Yong Tan S, Kan A, Nuchprayoon I, Jin R, Lee KH, et al: Expression of the FAT10 gene is highly upregulated in hepatocellular carcinoma and other gastrointestinal and gynecological cancers. Oncogene 22: 2592-2603, 2003.

19. Soucy TA, Dick LR, Smith PG, Milhollen MA and Brownell JE: The NEDD8 conjugation pathway and its relevance in cancer biology and therapy. Genes Cancer 1: 708-716, 2010.

20. Richardson PG, Sonneveld P, Schuster MW, Irwin D, Stadtmauer EA, Facon T, Harousseau JL, Ben-Yehuda D, Lonial S, Goldschmidt H, et al: Bortezomib or high-dose dexamethasone for relapsed multiple myeloma. N Engl J Med 352: 2487-2498, 2005.

21. San Miguel JF, Schlag R, Khuageva NK, Dimopoulos MA, Shpilberg O, Kropff M, Spicka I, Petrucci MT, Palumbo A, Samoilova OS, et al: Bortezomib plus melphalan and prednisone for initial treatment of multiple myeloma. N Engl J Med 359: 906-917, 2008.

22. Kane RC, Dagher R, Farrell A, Ko CW, Sridhara R, Justice R and Pazdur R: Bortezomib for the treatment of mantle cell lymphoma. Clin Cancer Res 13: 5291-5294, 2007. 
23. Yang Y, Kitagaki J, Dai RM, Tsai YC, Lorick KL, Ludwig RL, Pierre SA, Jensen JP, Davydov IV, Oberoi P, et al: Inhibitors of ubiquitin-activating enzyme (E1), a new class of potential cancer therapeutics. Cancer Res 67: 9472-9481, 2007.

24. Zhao Y, Xiong X, Jia L and Sun Y: Targeting Cullin-RING ligases by MLN4924 induces autophagy via modulating the HIF1-REDD1-TSC1-mTORC1-DEPTOR axis. Cell Death Dis 3: e386, 2012

25. Kamitani T, Kito K, Fukuda-Kamitani T and Yeh ET: Targeting of NEDD8 and its conjugates for proteasomal degradation by NUB1. J Biol Chem 276: 46655-46660, 2001.

26. Kito K, Yeh ET and Kamitani T: NUB1, a NEDD8-interacting protein, is induced by interferon and down-regulates the NEDD8 expression. J Biol Chem 276: 20603-20609, 2001.

27. Tanaka T, Kawashima H, Yeh ET and Kamitani T: Regulation of the NEDD8 conjugation system by a splicing variant, NUB1L. J Biol Chem 278: 32905-32913, 2003.

28. Tanji K, Tanaka T and Kamitani T: Interaction of NUB1 with the proteasome subunit S5a. Biochem Biophys Res Commun 337: $116-120,2005$

29. Groettrup M, Pelzer C, Schmidtke G and Hofmann K: Activating the ubiquitin family: UBA6 challenges the field. Trends Biochem Sci 33: 230-237, 2008.

30. Bornstein G, Ganoth D and Hershko A: Regulation of neddylation and deneddylation of cullin1 in SCFSkp2 ubiquitin ligase by F-box protein and substrate. Proc Natl Acad Sci USA 103 11515-11520, 2006.

31. Hosono T, Tanaka T, Tanji K, Nakatani T and Kamitani T: NUB1, an interferon-inducible protein, mediates anti-proliferative actions and apoptosis in renal cell carcinoma cells through cell-cycle regulation. Br J Cancer 102: 873-882, 2010.

32. Lu B, Al-Ramahi I, Valencia A, Wang Q, Berenshteyn F, Yang H, Gallego-Flores T, Ichcho S, Lacoste A, Hild M, et al: Identification of NUB1 as a suppressor of mutant Huntington toxicity via enhanced protein clearance. Nat Neurosci 16 : 562-570, 2013.

33. Kanaya K, Sohocki MM and Kamitani T: Abolished interaction of NUB1 with mutant AIPL1 involved in Leber congenital amaurosis. Biochem Biophys Res Commun 317: 768-773, 2004.

34. Tanaka $T$, Nakatani $T$ and Kamitani $T$ : Inhibition of NEDD8-conjugation pathway by novel molecules: Potential approaches to anticancer therapy. Mol Oncol 6: 267-275, 2012.

35. Haas AL, Ahrens P, Bright PM and Ankel H: Interferon induces a 15-kilodalton protein exhibiting marked homology to ubiquitin J Biol Chem 262: 11315-11323, 1987.

36. Ichimura Y, Kirisako T, Takao T, Satomi Y, Shimonishi Y, Ishihara N, Mizushima N, Tanida I, Kominami $\mathrm{E}$ Ohsumi M, et al: A ubiquitin-like system mediates protein lipidation. Nature 408: 488-492, 2000.

37. Rabut $G$ and Peter M: Function and regulation of protein neddylation. 'Protein modifications: Beyond the usual suspects' review series. EMBO Rep 9: 969-976, 2008.

38. Walden H, Podgorski MS, Huang DT, Miller DW, Howard RJ, Minor DL Jr, Holton JM and Schulman BA: The structure of the APPBP1-UBA3-NEDD8-ATP complex reveals the basis for selective ubiquitin-like protein activation by an E1. Mol Cell 12: 1427-1437, 2003.

39. Leidecker O, Matic I, Mahata B, Pion E and Xirodimas DP: The ubiquitin E1 enzyme Ube1 mediates NEDD8 activation under diverse stress conditions. Cell cycle 11: 1142-1150, 2012.

40. Olsen SK, Capili AD, Lu X, Tan DS and Lima CD: Active site remodelling accompanies thioester bond formation in the SUMO E1. Nature 463: 906-912, 2010

41. Huang DT, Hunt HW, Zhuang M, Ohi MD, Holton JM and Schulman BA: Basis for a ubiquitin-like protein thioester switch toggling E1-E2 affinity. Nature 445: 394-398, 2007.

42. Huang DT, Ayrault O, Hunt HW, Taherbhoy AM, Duda DM Scott DC, Borg LA, Neale G, Murray PJ, Roussel MF and Schulman BA: E2-RING expansion of the NEDD8 cascade confers specificity to cullin modification. Mol Cell 33: 483-495, 2009.

43. Kamura T, Conrad MN, Yan Q, Conaway RC and Conaway JW: The Rbx1 subunit of SCF and VHL E3 ubiquitin ligase activates Rub1 modification of cullins Cdc53 and Cul2. Genes Dev 13: 2928-2933, 1999.

44. Enchev RI,Schulman BA and Peter M: Protein neddylation: Beyond cullin-RING ligases. Nat Rev Mol Cell Biol 16: 30-44, 2015.

45. Hjerpe R, Thomas Y, Chen J, Zemla A, Curran S, Shpiro N, Dick LR and Kurz T: Changes in the ratio of free NEDD8 to ubiquitin triggers NEDDylation by ubiquitin enzymes. Biochem J 441: 927-936, 2012
46. Saha A and Deshaies RJ: Multimodal activation of the ubiquitin ligase SCF by Nedd8 conjugation. Mol Cell 32: 21-31, 2008.

47. Chan Y, Yoon J, Wu JT, Kim HJ, Pan KT, Yim J and Chien CT: DEN1 deneddylates non-cullin proteins in vivo. J Cell Sci 121: 3218-3223, 2008

48. Gong N, Li XY, Xiao Q and Wang YX: Identification of a novel spinal dorsal horn astroglial D-amino acid oxidase-hydrogen peroxide pathway involved in morphine antinociceptive tolerance. Anesthesiology 120: 962-975, 2014.

49. Lyapina S, Cope G, Shevchenko A, Serino G, Tsuge T, Zhou C, Wolf DA, Wei N, Shevchenko A and Deshaies RJ: Promotion of NEDD-CUL1 conjugate cleavage by COP9 signalosome. Science 292: 1382-1385, 2001.

50. Mendoza HM, Shen LN, Botting C, Lewis A, Chen J, Ink B and Hay RT: NEDP1, a highly conserved cysteine protease that deNEDDylates Cullins. J Biol Chem 278: 25637-25643, 2003.

51. Schwechheimer C, Serino G, Callis J, Crosby WL, Lyapina S, Deshaies RJ, Gray WM, Estelle M and Deng XW: Interactions of the COP9 signalosome with the E3 ubiquitin ligase SCFTIRI in mediating auxin response. Science 292: 1379-1382, 2001

52. Goldenberg SJ, Cascio TC, Shumway SD, Garbutt KC, Liu J, Xiong Y and Zheng N: Structure of the Cand1-Cul1-Roc1 complex reveals regulatory mechanisms for the assembly of the multisubunit cullin-dependent ubiquitin ligases. Cell 119: 517-528, 2004.

53. Liu J, Furukawa M, Matsumoto T and Xiong Y: NEDD8 modification of CUL1 dissociates p120 (CAND1), an inhibitor of CUL1-SKP1 binding and SCF ligases. Mol Cell 10: 1511-1518, 2002

54. Duda DM, Borg LA, Scott DC, Hunt HW, Hammel M and Schulman BA: Structural insights into NEDD8 activation of cullin-RING ligases: Conformational control of conjugation. Cell 134: 995-1006, 2008

55. Morimoto $M$, Nishida $T$, Nagayama $Y$ and Yasuda $H$ : Nedd8-modification of Cul1 is promoted by Roc1 as a Nedd8-E3 ligase and regulates its stability. Biochem Biophys Res Commun 301: 392-398, 2003

56. Osaka F, Kawasaki H, Aida N, Saeki M, Chiba T, Kawashima S, Tanaka K and Kato S: A new NEDD8-ligating system for cullin-4A. Genes Dev 12: 2263-2268, 1998.

57. Podust VN, Brownell JE, Gladysheva TB, Luo RS, Wang C, Coggins MB, Pierce JW, Lightcap ES and Chau V: A Nedd8 conjugation pathway is essential for proteolytic targeting of p27Kip1 by ubiquitination. Proc Natl Acad Sci USA 97: 4579-4584, 2000.

58. Read MA, Brownell JE, Gladysheva TB, Hottelet M, Parent LA, Coggins MB, Pierce JW, Podust VN, Luo RS, Chau V and Palombella VJ: Nedd8 modification of cul-1 activates SCF (beta (TrCP))-dependent ubiquitination of IkappaBalpha. Mol Cell Biol 20: 2326-2333, 2000.

59. Wada $\mathrm{H}$, Yeh ET and Kamitani T: Identification of NEDD8-conjugation site in human cullin-2. Biochem Biophys Res Commun 257: 100-105, 1999.

60. Kim W, Bennett EJ, Huttlin EL, Guo A, Li J, Possemato A, Sowa ME, Rad R, Rush J, Comb MJ, et al: Systematic and quantitative assessment of the ubiquitin-modified proteome. Mol Cell 44: 325-340, 2011.

61. Jeram SM, Srikumar T, Zhang XD, Anne Eisenhauer H, Rogers R, Pedrioli PG, Matunis M and Raught B: An improved SUMmOn-based methodology for the identification of ubiquitin and ubiquitin-like protein conjugation sites identifies novel ubiquitin-like protein chain linkages. Proteomics 10: 254-265, 2010.

62. van der Veen AG and Ploegh HL: Ubiquitin-like proteins. Annu Rev Biochem 81: 323-357, 2012.

63. McLarnon A: Cancer: Mdm2-regulated stabilization of HuR by neddylation in HCC and colon cancer-a possible target for therapy. Nat Rev Gastroenterol Hepatol 9: 4, 2011

64. Ryu JH, Li SH, Park HS, Park JW, Lee B and Chun YS: Hypoxia-inducible factor alpha subunit stabilization by NEDD8 conjugation is reactive oxygen species-dependent. J Biol Chem 286: 6963-6970, 2011.

65. Freiberg RA, Hammond EM, Dorie MJ, Welford SM and Giaccia AJ: DNA damage during reoxygenation elicits a Chk2-dependent checkpoint response. Mol Cell Biol 26: 1598-1609, 2006

66. Ma T, Chen Y, Zhang F, Yang CY, Wang S and Yu X: RNF111-dependent neddylation activates DNA damage-induced ubiquitination. Mol Cell 49: 897-907, 2013.

67. Le Moan N, Houslay DM, Christian F, Houslay MD and Akassoglou K: Oxygen-dependent cleavage of the p75 neurotrophin receptor triggers stabilization of HIF-1 $\alpha$. Mol Cell 44: 476-490, 2011 
68. Pawlus MR, Wang L and Hu CJ: STAT3 and HIF1 $\alpha$ cooperatively activate HIF1 target genes in MDA-MB-231 and RCC4 cells. Oncogene 33: 1670-1679, 2014

69. Choo YS, Vogler G, Wang D, Kalvakuri S, Iliuk A, Tao WA, Bodmer R and Zhang Z: Regulation of parkin and PINK1 by neddylation. Hum Mol Genet 21: 2514-2523, 2012.

70. Kandala S, Kim IM and Su H: Neddylation and deneddylation in cardiac biology. Am J Cardiovasc Dis 4: 140-158, 2014.

71. Abida WM, Nikolaev A, Zhao W, Zhang W and Gu W: FBXO11 promotes the Neddylation of $\mathrm{p} 53$ and inhibits its transcriptional activity. J Biol Chem 282: 1797-1804, 2007.

72. Noguchi K, Okumura F, Takahashi N, Kataoka A, Kamiyama T, Todo S and Hatakeyama S: TRIM40 promotes neddylation of IKK $\gamma$ and is downregulated in gastrointestinal cancers. Carcinogenesis 32: 995-1004, 2011.

73. Cao X and Sudhof TC: A transcriptionally [correction of transcriptively] active complex of APP with Fe65 and histone acetyltransferase Tip60. Science 293: 115-120, 2001.

74. Lee MR, Lee D, Shin SK, Kim YH and Choi CY: Inhibition of APP intracellular domain (AICD) transcriptional activity via covalent conjugation with Nedd8. Biochem Biophys Res Commun 366: 976-981, 2008.

75. Theng SS, Wang W, Mah WC, Chan C, Zhuo J, Gao Y, Qin H, Lim L, Chong SS, Song J and Lee CG: Disruption of FAT10-MAD2 binding inhibits tumor progression. Proc Natl Acad Sci USA 111: E5282-E5291, 2014.

76. Michel L, Diaz-Rodriguez E, Narayan G, Hernando E, Murty VV and Benezra R: Complete loss of the tumor suppressor MAD2 causes premature cyclin B degradation and mitotic failure in human somatic cells. Proc Natl Acad Sci USA 101: 4459-4464, 2004

77. Tapia C, Kutzner H, Mentzel T, Savic S, Baumhoer D and Glatz K: Two mitosis-specific antibodies, MPM-2 and phospho-histone H3 (Ser28), allow rapid and precise determination of mitotic activity. Am J Surg Pathol 30: 83-89, 2006.

78. Wagenaar-Miller RA, Gorden L and Matrisian LM: Matrix metalloproteinases in colorectal cancer: Is it worth talking about? Cancer Metastasis Rev 23: 119-135, 2004.

79. Aichem A, Kalveram B, Spinnenhirn V, Kluge K, Catone N, Johansen $\mathrm{T}$ and Groettrup $\mathrm{M}$ : The proteomic analysis of endogenous FAT10 substrates identifies p62/SQSTM1 as a substrate of FAT10ylation. J Cell Sci 125: 4576-4585, 2012.
80. Gao Y, Theng SS, Zhuo J, Teo WB, Ren J and Lee CG: FAT10, an ubiquitin-like protein, confers malignant properties in non-tumorigenic and tumorigenic cells. Carcinogenesis 35: 923-934, 2014

81. Lukasiak S, Schiller C, Oehlschlaeger P, Schmidtke G, Krause P, Legler DF, Autschbach F, Schirmacher P, Breuhahn K and Groettrup M: Proinflammatory cytokines cause FAT10 upregulation in cancers of liver and colon. Oncogene 27: 6068-6074, 2008.

82. Pelzer C, Kassner I, Matentzoglu K, Singh RK, Wollscheid HP, Scheffner M, Schmidtke G and Groettrup M: UBE1L2, a novel E1 enzyme specific for ubiquitin. J Biol Chem 282: 23010-23014, 2007.

83. Jin J, Li X, Gygi SP and Harper JW: Dual E1 activation systems for ubiquitin differentially regulate E2 enzyme charging. Nature 447: 1135-1138, 2007.

84. Chiu YH, Sun Q and Chen ZJ: E1-L2 activates both ubiquitin and FAT10. Mol Cell 27: 1014-1023, 2007.

85. Aichem A, Pelzer C, Lukasiak S, Kalveram B, Sheppard PW, Rani N, Schmidtke G and Groettrup M: USE1 is a bispecific conjugating enzyme for ubiquitin and FAT10, which FAT10ylates itself in cis. Nat Commun 1: 13, 2010

86. Hipp MS, Kalveram B, Raasi S, Groettrup M and Schmidtke G: FAT10, a ubiquitin-independent signal for proteasomal degradation. Mol Cell Biol 25: 3483-3491, 2005.

87. Hipp MS, Raasi S, Groettrup M and Schmidtke G: NEDD8 ultimate buster-1L interacts with the ubiquitin-like protein FAT10 and accelerates its degradation. J Biol Chem 279: 16503-16510, 2004.

88. Ren J, Kan A, Leong SH, Ooi LL, Jeang KT, Chong SS, Kon OL and Lee CG: FAT10 plays a role in the regulation of chromosomal stability. J Biol Chem 281: 11413-11421, 2006.

89. Merbl Y, Refour P, Patel H, Springer M and Kirschner MW: Profiling of ubiquitin-like modifications reveals features of mitotic control. Cell 152: 1160-1172, 2013.

90. Gong P, Canaan A, Wang B, Leventhal J, Snyder A, Nair V, Cohen CD, Kretzler M, D'Agati V, Weissman S and Ross MJ: The ubiquitin-like protein FAT10 mediates NF-kappaB activation. J Am Soc Nephrol 21: 316-326, 2010.

91. Bjorkoy G, Lamark T, Brech A, Outzen H, Perander M, Overvatn A, Stenmark H and Johansen T: p62/SQSTM1 forms protein aggregates degraded by autophagy and has a protective effect on huntingtin-induced cell death. J Cell Biol 171: 603-614, 2005. 